Article

\title{
On the Difference of Coefficients of Starlike and Convex Functions
}

\author{
Young Jae Sim ${ }^{1, *(1)}$ and Derek K. Thomas ${ }^{2}$ \\ 1 Department of Mathematics, Kyungsung University, Busan 48434, Korea \\ 2 Department of Mathematics, Swansea University, Bay Campus, Swansea SA1 8EN, UK; \\ d.k.thomas@swansea.ac.uk \\ * Correspondence: yjsim@ks.ac.kr
}

Received: 27 July 2020; Accepted: 2 September 2020; Published: 7 September 2020

\begin{abstract}
Let $f$ be analytic in the unit disk $\mathbb{D}=\{z \in \mathbb{C}:|z|<1\}$, and $\mathcal{S}$ be the subclass of normalized univalent functions given by $f(z)=z+\sum_{n=2}^{\infty} a_{n} z^{n}$ for $z \in \mathbb{D}$. Let $\mathcal{S}^{*} \subset \mathcal{S}$ be the subset of starlike functions in $\mathbb{D}$ and $\mathcal{C} \subset \mathcal{S}$ the subset of convex functions in $\mathbb{D}$. We give sharp upper and lower bounds for $\left|a_{3}\right|-\left|a_{2}\right|$ for some important subclasses of $\mathcal{S}^{*}$ and $\mathcal{C}$.
\end{abstract}

Keywords: univalent function; starlike function; convex function; difference of coefficients

\section{Introduction and Definitions}

Let $\mathcal{A}$ denote the class of analytic functions $f$ in the unit disk $\mathbb{D}=\{z \in \mathbb{C}:|z|<1\}$ normalized by $f(0)=0=f^{\prime}(0)-1$. Then for $z \in \mathbb{D}, f \in \mathcal{A}$ has the following representation

$$
f(z)=z+\sum_{n=2}^{\infty} a_{n} z^{n}
$$

Let $\mathcal{S}$ denote the subclass of all univalent (i.e., one-to-one) functions in $\mathcal{A}$.

In 1985, de Branges [1] solved the famous Bieberbach conjecture by showing that if $f \in \mathcal{S}$, then $\left|a_{n}\right| \leq n$ for $n \geq 2$, with equality when $f(z)=k(z):=z /(1-z)^{2}$, or a rotation. Therefore, it was natural to ask if for $f \in \mathcal{S}$, the inequality ||$a_{n+1}|-| a_{n}|| \leq 1$ is true when $n \geq 2$. This was shown not to be the case even when $n=2$ [2], and that the following sharp bounds hold.

$$
-1 \leq\left|a_{3}\right|-\left|a_{2}\right| \leq \frac{3}{4}+e^{-\lambda_{0}}\left(2 e^{-\lambda_{0}}-1\right)=1.029 \cdots,
$$

where $\lambda_{0}$ is the unique value of $\lambda$ in $0<\lambda<1$, satisfying the equation $4 \lambda=e^{\lambda}$.

Hayman [3] showed that if $f \in \mathcal{S}$, then ||$a_{n+1}|-| a_{n}|| \leq C$, where $C$ is an absolute constant. The exact value of $C$ is unknown, the best estimate to date being $C=3.61 \cdots[4]$, which because of the sharp estimate above when $n=2$, cannot be reduced to 1 .

Hayman's seminal result ||$a_{n+1}|-| a_{n}|| \leq C$ for $n \geq 2$, was proved in 1963, using his distinctive method developed to study areally mean $p$-valent functions. A different proof was provided by Milin, using the now well-known Lebedev-Milin inequalities, and an excellent account of this result can be found in Duren's book [2]. Little progress has been made estimating the value of $C$. It was shown by Ilina [5] in 
1968 that $C<4.26 \ldots$, which, using a modification of Milin's method, Grispan [4] improved in 1976 to show that for $n \geq 2$,

$$
-2.97 \cdots<\left|a_{n+1}\right|-\left|a_{n}\right|<3.61 \ldots
$$

No other advances appear to have been made in this direction during the intervening years, until a recent result of Obradović, Thomas and Tuneski [6], who, using the Grunsky inequalities, have shown that the upper bound for $C$ can be improved when $n=3$ to $\left|a_{4}\right|-\left|a_{3}\right| \leq 2.1033 \ldots$.

Thus, apart from the inequalities for $n=2$ above, there appears to be no known sharp upper or lower bounds for $\left|a_{n+1}\right|-\left|a_{n}\right|$ when $n \geq 3$ for functions in $\mathcal{S}$.

Definition 1. Let $f \in \mathcal{A}$ and be given by (1). Denote by $\mathcal{S}^{*}$ the subclass of $\mathcal{A}$ consisting of starlike functions, i.e., functions $f$ which map $\mathbb{D}$ onto a set which is star-shaped with respect to the origin. Subsequently, it is well-known that a function $f \in \mathcal{S}^{*}$ if, and only if, for $z \in \mathbb{D}$

$$
\operatorname{Re}\left\{\frac{z f^{\prime}(z)}{f(z)}\right\}>0
$$

Without doubt the most complete solution to finding sharp bounds for the difference of successive coefficients is the following theorem of Leung [7], who, in 1978, proved the sharp inequality ||$a_{n+1} \mid-$ $\left|a_{n}\right| \mid \leq 1$ for $n \geq 2$, when $f \in \mathcal{S}^{*}$, the proof of which relies on a lemma concerning functions with positive real, and the third Lebedev-Milin inequality.

Theorem 1. Let $f \in \mathcal{S}^{*}$ and be given by (1). Subsequently, ||$a_{n+1}|-| a_{n}|| \leq 1$ for $n \geq 2$ with equality for functions of the type $z /((1-\gamma z)(1-\zeta z))$, for some $\gamma$ and $\zeta$, with $|\gamma|=|\zeta|=1$.

The question therefore arises as to whether there are analogous sharp bounds for ||$a_{n+1}|-| a_{n}||$ for the more significant subclasses of subclasses of $\mathcal{S}^{*}$ such as starlike functions of order $\alpha$, etc. By considering the case $n=2$, we will demonstrate that no such single bounds exist, by finding distinct sharp lower and upper bounds for the classes of starlike functions of order $\alpha$, and for the class of strongly starlike functions.

We will also demonstrate that a similar situation holds for subclasses of the convex functions, which we define as follows.

Definition 2. Let $f \in \mathcal{A}$ and be given by (1). Denote, by $\mathcal{C}$, the subclass of $\mathcal{A}$ consisting of convex functions, i.e., functions $f$ which map $\mathbb{D}$ onto a set which is convex. Subsequently, it is well-known that a function $f \in \mathcal{C}$ if, and only if, for $z \in \mathbb{D}$.

$$
\operatorname{Re}\left\{1+\frac{z f^{\prime \prime}(z)}{f^{\prime}(z)}\right\}>0 .
$$

Thus $f \in \mathcal{C}$ if, and only if, $z f^{\prime} \in \mathcal{S}^{*}$ for $z \in \mathbb{D}$.

We next define what we consider to be the most significant subclasses of $\mathcal{S}^{*}$ and $\mathcal{C}$, as follows.

Definition 3. Let $\mathcal{A}$ and be given by (1). Denote by $\mathcal{S}^{*}(\alpha)$ the subclass of $\mathcal{S}^{*}$ consisting of starlike functions of order $\alpha$ i.e., for $0 \leq \alpha<1$ and $z \in \mathbb{D}$,

$$
\operatorname{Re}\left\{\frac{z f^{\prime}(z)}{f(z)}\right\}>\alpha .
$$


Definition 4. Let $\mathcal{A}$ and be given by (1). Denote, by $\mathcal{C}(\alpha)$, the subclass of $\mathcal{C}$ consisting of convex functions of order $\alpha$ i.e., for $0 \leq \alpha<1$ and $z \in \mathbb{D}$,

$$
\operatorname{Re}\left\{1+\frac{z f^{\prime \prime}(z)}{f^{\prime}(z)}\right\}>\alpha
$$

We next recall the class $\mathcal{S}^{* *}(\alpha)$ of strongly starlike functions of order $\alpha$, defined as follows.

Definition 5. Let $\mathcal{A}$ and be given by (1). Denote, by $\mathcal{S}^{* *}(\alpha)$, the subclass of $\mathcal{S}^{*}$ consisting of strongly starlike functions order $\alpha$ i.e., for $0<\alpha \leq 1$ and $z \in \mathbb{D}$,

$$
\left|\arg \frac{z f^{\prime}(z)}{f(z)}\right|<\frac{\alpha \pi}{2}
$$

We finally define the class $\mathcal{C}^{* *}(\alpha)$ of strongly convex functions of order $\alpha$, as follows.

Definition 6. Let $\mathcal{A}$ and be given by (1). Denote, by $\mathcal{C}^{* *}(\alpha)$, the subclass of $\mathcal{C}$ consisting of strongly convex functions order $\alpha$ i.e., for $0<\alpha \leq 1$ and $z \in \mathbb{D}$,

$$
\left|\arg \left(1+\frac{z f^{\prime \prime}(z)}{f^{\prime}(z)}\right)\right|<\frac{\alpha \pi}{2}
$$

We note at this point, that even though the problem of finding sharp upper and lower bounds $\left|a_{n+1}\right|-\left|a_{n}\right|$ is solved for $n \geq 2$, [7] when $f \in \mathcal{S}^{*}$, obtaining sharp lower bounds for $\left|a_{n+1}\right|-\left|a_{n}\right|$, for $n \geq 2$ when $f \in \mathcal{C}(0)$ appears to be a difficult problem. Sharp upper bounds for $n \geq 2$ follow at once from the starlike case, and the fact that $f \in \mathcal{C}$ if, and only if $z f^{\prime} \in \mathcal{S}^{*}$. The only sharp lower bounds to date are due to Ming and Sugawa [8], for $n=2$ and 3. Thus when $n \geq 4$, finding sharp lower bounds for $f \in \mathcal{C}$ remains an open problem.

\section{Preliminary Lemmas}

Denote, by $\mathcal{P}$, the class of analytic functions $p$ with positive real part on $\mathbb{D}$ given by

$$
p(z)=1+\sum_{n=1}^{\infty} p_{n} z^{n}
$$

We will use the following lemmas for the coefficients of functions $\mathcal{P}$, given by (3).

Lemma 1 ([2], p. 41). For $p \in \mathcal{P},\left|p_{n}\right| \leq 2$ for $n \geq 1$. The inequalities are sharp.

Lemma 2 ([9]). If $p \in \mathcal{P}$, then

$$
\begin{aligned}
& p_{1}=2 \zeta_{1}, \\
& p_{2}=2 \zeta_{1}^{2}+2\left(1-\left|\zeta_{1}\right|^{2}\right) \zeta_{2}
\end{aligned}
$$

for some $\zeta_{i} \in \overline{\mathbb{D}}, i \in\{1,2\}$.

For $\zeta_{1} \in \mathbb{T}$, the boundary of $\mathbb{D}$, there is a unique function $p \in \mathcal{P}$ with $p_{1}$ as above, namely,

$$
p(z)=\frac{1+\zeta_{1} z}{1-\zeta_{1} z} \quad(z \in \mathbb{D}) .
$$


For $\zeta_{1} \in \mathbb{D}$ and $\zeta_{2} \in \mathbb{T}$, there is a unique function $p \in \mathcal{P}$ with $p_{1}$ and $p_{2}$ as above, namely,

$$
p(z)=\frac{1+\left(\bar{\zeta}_{1} \zeta_{2}+\zeta_{1}\right) z+\zeta_{2} z^{2}}{1+\left(\bar{\zeta}_{1} \zeta_{2}-\zeta_{1}\right) z-\zeta_{2} z^{2}} \quad(z \in \mathbb{D})
$$

Lemma 3 ([10]). Suppose that $p \in \mathcal{P}$, with coefficients given by (3), and $p_{1} \geq 0$.

Then for some complex-valued $y$ with $|y| \leq 1$

$$
2 p_{2}=p_{1}^{2}+y\left(4-p_{1}^{2}\right)
$$

We note at the outset that all of the following classes of functions, and the functional $\left|a_{3}\right|-\left|a_{2}\right|$ are rotationally invariant. We also note that the proofs of the following theorems are similar, and so we omit some of the details for brevity.

\section{Coefficient Differences for $\mathcal{S}^{*}(\alpha)$}

We prove the following, noting that when $\alpha=0$, we obtain the result in [7].

Theorem 2. Let $f \in \mathcal{S}^{*}(\alpha)$ and be given by (1). Subsequently, for $0 \leq \alpha<1$,

$$
-\frac{\sqrt{2}(1-\alpha)}{\sqrt{2-\alpha}} \leq\left|a_{3}\right|-\left|a_{2}\right| \leq 1-\alpha .
$$

Both inequalities are sharp.

Proof. We begin by noting that if $f \in \mathcal{S}^{*}(\alpha)$, then, from (2), we can write

$$
z f^{\prime}(z)=f(z)(\alpha+(1-\alpha) p(z))
$$

for some $p \in \mathcal{P}$.

Equating coefficients gives

$$
\begin{aligned}
& a_{2}=(1-\alpha) p_{1} \\
& a_{3}=\frac{(1-\alpha)}{2}\left(p_{2}+(1-\alpha) p_{1}^{2}\right),
\end{aligned}
$$

and so after a rotation so that $p_{1}=p$, with $0 \leq p \leq 2$ we obtain, using Lemma 3 and the fact that $|y| \leq 1$,

$$
\begin{aligned}
\left|a_{3}\right|-\left|a_{2}\right| & =\frac{(1-\alpha)}{2}\left|(1-\alpha) p^{2}+\frac{1}{2}\left(p^{2}+\left(4-p^{2}\right) y\right)\right|-(1-\alpha) p \\
& \leq \frac{(1-\alpha)}{2}\left[(1-\alpha) p^{2}+\frac{1}{2}\left(p^{2}+\left(4-p^{2}\right)\right)\right]-(1-\alpha) p \\
& =\frac{1}{2}(1-\alpha)\left(2+(1-\alpha) p^{2}\right)-(1-\alpha) p .
\end{aligned}
$$

It is now a simple exercise to show that the maximum value of the above expression when $0 \leq \alpha<1$ and $0 \leq p \leq 2$ is $1-\alpha$, which gives the upper bound.

For the lower bound we use Lemma 2, which gives

$$
\left|a_{2}\right|-\left|a_{3}\right|=2(1-\alpha)\left|\zeta_{1}\right|-\frac{1-\alpha}{2}\left|2 \zeta_{1}^{2}+4(1-\alpha) \zeta_{1}^{2}+2 \zeta_{2}\left(1-\left|\zeta_{1}\right|^{2}\right)\right| .
$$


After a rotation, we can assume that $\zeta_{1}=t$, where $0 \leq t \leq 1$ and so writing $\zeta_{2}=s e^{i \phi}$ with $0 \leq s \leq 1$ and $0 \leq \phi \leq 2 \pi$, we obtain

$$
\begin{aligned}
\left|a_{2}\right|-\left|a_{3}\right| & =2(1-\alpha) t-(1-\alpha)\left|(3-2 \alpha) t^{2}+s e^{i \phi}\left(1-t^{2}\right)\right| \\
& =2(1-\alpha) t-(1-\alpha) \sqrt{\left((3-2 \alpha) t^{2}\right)^{2}+\left(s\left(1-t^{2}\right)\right)^{2}+2(3-2 \alpha) t^{2} s\left(1-t^{2}\right) \cos [\phi]} \\
& \leq 2(1-\alpha) t-(1-\alpha)\left|(3-2 \alpha) t^{2}-s\left(1-t^{2}\right)\right|=(1-\alpha) \Phi_{1}(t, s),
\end{aligned}
$$

where $\Phi_{1}$ is defined by

$$
\Phi_{1}(t, s)=2 t-\left|(3-2 \alpha) t^{2}-s\left(1-t^{2}\right)\right| .
$$

When $0 \leq t \leq \frac{1}{\sqrt{4-2 \alpha}}$, we have

$$
\Phi_{1}(t, s) \leq 2 t \leq \frac{\sqrt{2}}{\sqrt{2-\alpha}}
$$

If $\frac{1}{\sqrt{4-2 \alpha}} \leq t \leq 1$, then $(3-2 \alpha) t^{2}-s\left(1-t^{2}\right) \geq 0$ for all $s \in[0,1]$, since

$$
(3-2 \alpha) t^{2}-s\left(1-t^{2}\right)=(3-2 \alpha+s) t^{2}-s \geq \frac{(3-2 \alpha)(1-s)}{4-2 \alpha} \geq 0, \quad s \in[0,1] .
$$

Thus, we have

$$
\Phi_{1}(t, s)=2 t-(3-2 \alpha) t^{2}+s\left(1-t^{2}\right) \leq-2(2-\alpha) t^{2}+2 t+1=: g_{1}(t),
$$

and

$$
g_{1}(t) \leq g_{1}\left(\frac{1}{\sqrt{4-2 \alpha}}\right)=\frac{\sqrt{2}}{\sqrt{2-\alpha}}
$$

which implies that

$$
\Phi_{1}(t, s) \leq \frac{\sqrt{2}}{\sqrt{2-\alpha}}
$$

Hence, from (5), (6) and (4), we obtain the required lower bound.

The upper bound is sharp when $f \in \mathcal{A}$ is given by

$$
f(z)=\frac{z}{\left(1-z^{2}\right)^{1-\alpha}}
$$

and the lower bound is sharp when $f \in \mathcal{A}$ is given by

$$
f(z)=z \exp \left[2(1-\alpha) \int_{0}^{z} \frac{t_{0}-\xi}{1-2 t_{0} \xi+\xi^{2}} d \xi\right],
$$

where $t_{0}=1 /(\sqrt{2} \sqrt{2-\alpha})$.

\section{Coefficient Differences for $\mathcal{C}(\alpha)$}

We prove the following, noting that, when $\alpha=0$, gives the inequalities obtained in [8]. 
Theorem 3. Let $f \in \mathcal{C}(\alpha)$ and be given by (1). Subsequently, for $0 \leq \alpha<1$,

$$
\left|a_{3}\right|-\left|a_{2}\right| \leq \frac{(1-\alpha)}{3}
$$

and

$$
\left|a_{3}\right|-\left|a_{2}\right| \geq \begin{cases}\frac{(1-\alpha)}{\sqrt{2} \sqrt{2-\alpha},} & \text { when } 0 \leq \alpha \leq 7 / 8 \\ \frac{(1-\alpha)(25-8 \alpha)}{24(2-\alpha)}, & \text { when } 7 / 8 \leq \alpha<1 .\end{cases}
$$

All of the inequalities are sharp.

Proof. Because $f \in \mathcal{C}(\alpha)$ if, and only if, $z f^{\prime} \in \mathcal{S}^{*}(\alpha)$, the upper bound follows at once from Theorem 2 on noting that, since $3\left|a_{3}\right|-2\left|a_{2}\right| \leq 1-\alpha$, we obtain

$$
\left|a_{3}\right|-\left|a_{2}\right| \leq\left|a_{3}\right|-\frac{2}{3}\left|a_{2}\right| \leq \frac{1-\alpha}{3} .
$$

For the lower bound, we proceed as in Theorem 2, again use Lemma 2, and after equating coefficients and making a rotation, arrive at the expression

$$
\begin{aligned}
\left|a_{2}\right|-\left|a_{3}\right| & =(1-\alpha) t-\frac{1}{6}(1-\alpha)\left|2(3-2 \alpha) t^{2}+2 s e^{i \phi}\left(1-t^{2}\right)\right| \\
& \leq(1-\alpha) t-\frac{1}{3}(1-\alpha)\left|(3-2 \alpha) t^{2}-s\left(1-t^{2}\right)\right|=(1-\alpha) \Phi_{2}(t, s)
\end{aligned}
$$

where $\Phi_{2}$ is defined by

$$
\Phi_{2}(t, s)=t-\frac{1}{3}\left|(3-2 \alpha) t^{2}-s\left(1-t^{2}\right)\right|
$$

When $0 \leq t \leq \frac{1}{\sqrt{2} \sqrt{2-\alpha}}$, we have

$$
\Phi_{2}(t, s) \leq t \leq \frac{1}{\sqrt{2} \sqrt{2-\alpha}}
$$

If $\frac{1}{\sqrt{2} \sqrt{2-\alpha}} \leq t \leq 1$, then $(3-2 \alpha) t^{2}-s\left(1-t^{2}\right) \geq 0$ for all $s \in[0,1]$, and so

$$
\Phi_{2}(t, s)=-\frac{1}{3}(3-2 \alpha) t^{2}+t+\frac{1}{3}\left(1-t^{2}\right) s \leq-\frac{1}{3}(4-2 \alpha) t^{2}+t+\frac{1}{3}=: g_{2}(t) .
$$

When $0 \leq \alpha \leq \frac{7}{8}$, the function $g_{2}$ is decreasing on $\left[\frac{\sqrt{2}}{\sqrt{2-\alpha}}, 1\right]$, and so

$$
\Phi_{2}(t, s) \leq g_{2}\left(\frac{1}{\sqrt{2} \sqrt{2-\alpha}}\right)=\frac{1}{\sqrt{2} \sqrt{2-\alpha}}
$$

When $\frac{7}{8} \leq \alpha<1, g_{2}^{\prime}(t)=0$ at $t=t_{0}:=\frac{3}{4(2-\alpha)}$, and so

$$
\Phi_{2}(t, s) \leq g_{2}\left(t_{0}\right)=\frac{25-8 \alpha}{24(2-\alpha)}
$$


Thus, from (8)-(10), it follows that

$$
\Phi_{2}(t, s) \leq \begin{cases}\frac{1}{\sqrt{2} \sqrt{2-\alpha},} & \text { if } 0 \leq \alpha \leq 7 / 8, \\ \frac{25-8 \alpha}{24(2-\alpha)}, & \text { if } 7 / 8 \leq \alpha<1,\end{cases}
$$

for all $(t, s) \in[0,1] \times[0,1]$, which by $(7)$, gives the required lower bound.

The bound $\left|a_{3}\right|-\left|a_{2}\right|$ is sharp when $f \in \mathcal{A}$ is given by

$$
f(z)=\int_{0}^{z} \frac{d \xi}{\left(1-\xi^{2}\right)^{1-\alpha}}
$$

and the first lower bound for $\left|a_{2}\right|-\left|a_{3}\right|$ is sharp when $f \in \mathcal{A}$ is given by

$$
f(z)=\int_{0}^{z} \exp \left[2(1-\alpha) \int_{0}^{\eta} \frac{t_{0}-\xi}{1-2 t_{0} \xi+\xi^{2}} d \xi\right] d \eta
$$

where $t_{0}=1 /(\sqrt{2} \sqrt{2-\alpha})$, and $t_{0}=3 /(4(2-\alpha))$ for the second bound.

\section{Coefficient Differences for $\mathcal{S}^{* *}(\alpha)$}

Theorem 4. Let $f \in \mathcal{S}^{* *}(\alpha)$ and be given by (1). Subsequently, for $0<\alpha \leq 1$,

$$
-\frac{2 \alpha}{\sqrt{3 \alpha+1}} \leq\left|a_{3}\right|-\left|a_{2}\right| \leq \alpha
$$

Both the inequalities are sharp.

Proof. Note first note that from (5), we can write

$$
\frac{z f^{\prime}(z)}{f(z)}=p(z)^{\alpha}
$$

for some $p \in \mathcal{P}$, and so equating coefficients we obtain, using Lemma 2,

$$
\begin{aligned}
& a_{2}=\alpha p_{1}=2 \alpha \zeta_{1}, \\
& a_{3}=\frac{\alpha}{4}\left((3 \alpha-1) p_{1}^{2}+2 p_{2}\right)=\alpha\left(\zeta_{1}^{2}-(1-3 \alpha) \zeta_{1}^{2}+\zeta_{2}\left(1-\left|\zeta_{1}\right|^{2}\right)\right) .
\end{aligned}
$$

We begin by proving the upper bound. After a rotation, noting that from Lemma 1 that $\left|p_{1}\right| \leq 2$, we can assume that $p_{1}=p$, with $0 \leq p \leq 2$, and using Lemma 3 obtain, since $|y| \leq 1$,

$$
\begin{aligned}
\left|a_{3}\right|-\left|a_{2}\right| & =\frac{\alpha}{2}\left|p_{2}-\frac{1}{2} p^{2}(1-3 \alpha)\right|-\alpha p, \\
& =\frac{\alpha}{2}\left|\frac{1}{2}\left(\left(4-p^{2}\right) y+\frac{3 p^{2} \alpha}{2}\right)\right|-\alpha p, \\
& \leq \frac{\alpha}{2}\left[\frac{1}{2}\left(\left(4-p^{2}\right)+\frac{3 p^{2} \alpha}{2}\right)\right]-\alpha p .
\end{aligned}
$$


Elementary calculus shows that the above expression has a minimum at $p=2 /(3 \alpha-1)$, and that the maximum value occurs at $p=0$, which gives the required upper bound.

Using Lemma 2, we next establish the lower bound in Theorem 4.

Writing $\zeta_{1}=t$ and $\zeta_{2}=s e^{i \phi}$, so that $0 \leq t \leq 1,0 \leq s \leq 1$, and $0 \leq \phi \leq 2 \pi$, using Lemma 2 and (4), we can write

$$
\begin{aligned}
\left|a_{2}\right|-\left|a_{3}\right| & =2 t \alpha-\frac{1}{2} \alpha\left|6 t^{2} \alpha+2 s e^{i \phi}\left(1-t^{2}\right)\right| \\
& =2 t \alpha-\frac{1}{2} \alpha \sqrt{\left(6 t^{2} \alpha\right)^{2}+\left(2 s\left(1-t^{2}\right)\right)^{2}+2\left(6 t^{2} \alpha\right)\left(2 s\left(1-t^{2}\right)\right) \cos \phi} \\
& \leq 2 t \alpha-\frac{1}{2} \alpha\left|6 t^{2} \alpha-2 s\left(1-t^{2}\right)\right|=\alpha \Phi_{3}(t, s),
\end{aligned}
$$

where $\Phi_{3}$ is defined by

$$
\Phi_{3}(t, s)=2 t-\left|3 t^{2} \alpha-s\left(1-t^{2}\right)\right| .
$$

When $0 \leq t \leq \frac{1}{\sqrt{3 \alpha+1}}$, we have

$$
\Phi_{3}(t, s) \leq 2 t \leq \frac{1}{\sqrt{3 \alpha+1}}
$$

If $\frac{1}{\sqrt{3 \alpha+1}} \leq t \leq 1$, then $3 \alpha t^{2}-s\left(1-t^{2}\right) \geq 0$ for all $s \in[0,1]$, since

$$
3 \alpha t^{2}-s\left(1-t^{2}\right)=(3 \alpha+s) t^{2}-s \geq \frac{3 \alpha(1-s)}{3 \alpha+1} \geq 0, \quad s \in[0,1]
$$

Thus,

$$
\Phi_{3}(t, s)=2 t-3 \alpha t^{2}+s\left(1-t^{2}\right) \leq-(3 \alpha+1) t^{2}+2 t+1=: g_{3}(t),
$$

and

$$
g_{3}(t) \leq g_{3}\left(\frac{1}{\sqrt{3 \alpha+1}}\right)=\frac{2}{\sqrt{3 \alpha+1}}
$$

which implies

$$
\Phi_{3}(t, s) \leq \frac{2}{\sqrt{3 \alpha+1}} .
$$

Thus, from (13), (14), and (12), we obtain the required lower bound.

The upper bound is sharp when $f \in \mathcal{A}$ is given by

$$
f(z)=z \exp \left[\int_{0}^{z} \frac{1}{\xi}\left(\left(\frac{1+\xi^{2}}{1-\xi^{2}}\right)^{\alpha}-1\right) d \xi\right],
$$

and the lower bound is sharp when $f \in \mathcal{A}$ is given by

$$
f(z)=z \exp \left[\int_{0}^{z} \frac{1}{\xi}\left(\left(\frac{1-\xi^{2}}{1-t_{0} \xi^{2}+\xi^{2}}\right)^{\alpha}-1\right) d \xi\right]
$$

where $t_{0}=1 / \sqrt{1+3 \alpha}$. 


\section{Coefficient Differences for $\mathcal{C}^{* *}(\alpha)$}

We prove the following.

Theorem 5. Let $f \in \mathcal{C}^{* *}(\alpha)$ and be given by (1). Subsequently, for $0<\alpha \leq 1$,

$$
\left|a_{3}\right|-\left|a_{2}\right| \leq \frac{\alpha}{3}
$$

Also,

$$
\left|a_{2}\right|-\left|a_{3}\right| \leq \begin{cases}\alpha(1-\alpha), & \text { when } 0<\alpha \leq \frac{1}{6} \\ \frac{\alpha(13+12 \alpha)}{12(1+3 \alpha)}, & \text { when } \frac{1}{6} \leq \alpha \leq \frac{5}{12} \\ \frac{\alpha}{\sqrt{1+3 \alpha}}, & \text { when } \frac{5}{12} \leq \alpha \leq 1\end{cases}
$$

All the inequalities are sharp.

Proof. We proceed as in Theorem 4, first noticing that since $f \in \mathcal{C}^{* *}(\alpha)$ if, and only if, $z f^{\prime} \in \mathcal{S}^{* *}(\alpha)$, the upper bound follows at once, as in Theorem 2.

For the lower bound, we again follow the method in Theorem 4, to obtain after equating coefficients, applying Lemma 2, and making a rotation, arrive at the following expression.

$$
\begin{aligned}
\left|a_{2}\right|-\left|a_{3}\right| & =t \alpha-\frac{1}{3} \alpha\left|3 t^{2} \alpha+s e^{i \phi}\left(1-t^{2}\right)\right| \\
& =t \alpha-\frac{1}{3} \alpha \sqrt{\left(3 t^{2} \alpha\right)^{2}+\left(s\left(1-t^{2}\right)\right)^{2}+2\left(3 t^{2} \alpha\right)\left(s\left(1-t^{2}\right)\right) \cos \phi} \\
& \leq t \alpha-\frac{1}{3} \alpha\left|3 t^{2} \alpha-s\left(1-t^{2}\right)\right|=\alpha \Phi_{4}(t, s),
\end{aligned}
$$

where $\Phi_{4}$ is defined by

$$
\Phi_{4}(t, s)=t-\frac{1}{3}\left|3 t^{2} \alpha-s\left(1-t^{2}\right)\right| .
$$

When $0 \leq t \leq \frac{1}{\sqrt{3 \alpha+1}}$, we have

$$
\Phi_{4}(t, s) \leq t \leq \frac{1}{\sqrt{3 \alpha+1}}
$$

If $\frac{1}{\sqrt{3 \alpha+1}} \leq t \leq 1$, then $3 \alpha t^{2}-s\left(1-t^{2}\right) \geq 0$ for all $s \in[0,1]$, and so

$$
\Phi_{4}(t, s)=t-\alpha t^{2}+\frac{1}{3} s\left(1-t^{2}\right) \leq-\left(\frac{1}{3}+\alpha\right) t^{2}+t+\frac{1}{3}=: g_{4}(t)
$$

When $0<\alpha \leq \frac{1}{6}$, the function $g_{4}$ is increasing on $\left[\frac{1}{\sqrt{3 \alpha+1}}, 1\right]$, and so

$$
\Phi_{4}(t, s) \leq g_{4}(1)=1-\alpha .
$$




$$
\begin{aligned}
& \text { If } \frac{1}{6} \leq \alpha \leq \frac{5}{12}, g_{4}^{\prime}(t)=0 \text { at } t=t_{0}:=\frac{3}{2(1+3 \alpha)} \text {, and so } \\
& \qquad \Phi_{4}(t, s) \leq g_{4}\left(t_{0}\right)=\frac{13+12 \alpha}{12(1+3 \alpha)} .
\end{aligned}
$$

If $\frac{5}{12} \leq \alpha<1$, the function $g_{4}$ is decreasing on $\left[\frac{1}{\sqrt{3 \alpha+1}}, 1\right]$, and so

$$
\Phi_{4}(t, s) \leq g_{4}\left(\frac{1}{\sqrt{3 \alpha+1}}\right)=\frac{1}{\sqrt{3 \alpha+1}}
$$

Thus, from (16)-(19), we have

$$
\Phi_{4}(t, s) \leq \begin{cases}(1-\alpha), & \text { if } 0<\alpha \leq \frac{1}{6} \\ \frac{13+12 \alpha}{12(1+3 \alpha)}, & \text { if } 1 / 6 \leq \alpha \leq \frac{5}{12} \\ \frac{1}{\sqrt{3 \alpha+1}}, & \text { if } \frac{5}{12} \leq \alpha \leq 1\end{cases}
$$

for all $(t, s) \in[0,1] \times[0,1]$, which from (15), gives the required lower bound.

The first inequality in Theorem 5 is sharp when $f \in \mathcal{A}$ is given by

$$
f(z)=\int_{0}^{z} \exp \left[\int_{0}^{\eta} \frac{1}{\xi}\left(\left(\frac{1+\xi}{1-\xi}\right)^{\alpha}-1\right) d \xi\right] d \eta
$$

the second inequality is sharp when

$$
f(z)=\int_{0}^{z} \exp \left[\int_{0}^{\eta} \frac{1}{\xi}\left(\left(\frac{1-\xi^{2}}{1-2 t_{1} \xi+\xi^{2}}\right)^{\alpha}-1\right) d \xi\right] d \eta
$$

with $t_{1}=3 /(2+3 \alpha)$, and the third inequality is sharp when

$$
f(z)=\int_{0}^{z} \exp \left[\int_{0}^{\eta} \frac{1}{\xi}\left(\left(\frac{1-\xi^{2}}{1-2 t_{2} \xi+\xi^{2}}\right)^{\alpha}-1\right) d \xi\right] d \eta,
$$

with $t_{2}=1 / \sqrt{1+3 \alpha}$

Author Contributions: Writing—original draft, Y.J.S. and D.K.T.; Writing—review and editing, Y.J.S. and D.K.T. All authors have read and agreed to the published version of the manuscript.

Funding: The first author was supported by the National Research Foundation of Korea (NRF) grant funded by the Korea government (MSIP; Ministry of Science, ICT \& Future Planning) (No. NRF-2017R1C1B5076778).

Conflicts of Interest: The authors declare no conflict of interest.

\section{References}

1. Branges, L.D. A proof of the Bieberbach conjecture. Acta Math. 1985, 154, 137-152. [CrossRef]

2. Duren, P.L. Univalent Functions; Springer: New York, NY, USA, 1983.

3. Hayman, W.K. On successive coefficients of univalent functions. J. Lond. Math. Soc. 1963, 38, 228-243. [CrossRef] 
4. Grinspan, A.Z. The sharpening of the difference of the moduli of adjacent coefficients of schlicht functions. In Problems in Modern Function Theory, Proceedings of the Conference on Modern Problems of the Geometric Theory of Functions; Institute of Mathematics, Siberian Branch, Academy of Sciences of the USSR: Novosibirsk, Russia, 1976; pp. 41-45.

5. Ilina, L.P. The relative growth of nearby coefficients of schlicht functions. Mat. Zametki 1968, 4, 715-722.

6. Obradović, M.; Thomas, D.K.; Tuneski, N. On the difference of coefficients of univalent functions. arXiv 2020, arXiv:2004.06369.

7. Leung, Y. Successive coefficients of starlike functions. Bull. Lond. Math. Soc. 1978, 10, 193-196. [CrossRef]

8. Ming, L.; Sugawa, T. A Note on Successive Coefficients of Convex Functions. Comput. Methods Funct. Theory 2016, 17. [CrossRef]

9. Cho, N.E.; Kowalczyk, B.; Lecko, A. The sharp bounds of some coefficient functionals over the class of functions convex in the direction of the imaginary axis. Bull. Aust. Math. Soc. 2019, 100, 86-96. [CrossRef]

10. Libera, R.J.; Złotkiewicz, E.J. Coefficient bounds for the inverse of a function with derivatives in $\mathcal{P}$. Proc. Am. Math. Soc. 1983, 87, 251-257. [CrossRef]

(C) 2020 by the authors. Licensee MDPI, Basel, Switzerland. This article is an open access article distributed under the terms and conditions of the Creative Commons Attribution (CC BY) license (http:/ / creativecommons.org/licenses/by/4.0/). 